\title{
Orientation Histograms as Shape Priors for Left Ventricle Segmentation Using Graph Cuts
}

\author{
Dwarikanath Mahapatra and Ying Sun \\ Department of Electrical and Computer Engineering, \\ National University of Singapore, 4 Engineering Drive 3, Singapore 117576 \\ dmahapatra@gmail.com, elesuny@nus.edu.sg
}

\begin{abstract}
Poor contrast in magnetic resonance images makes cardiac left ventricle $(\mathrm{LV})$ segmentation a very challenging task. We propose a novel graph cut framework using shape priors for segmentation of the LV from dynamic cardiac perfusion images. The shape prior information is obtained from a single image clearly showing the LV. The shape penalty is assigned based on the orientation angles between a pixel and all edge points of the prior shape. We observe that the orientation angles have distinctly different distributions for points inside and outside the LV. To account for shape change due to deformations, pixels near the boundary of the prior shape are allowed to change their labels by appropriate formulation of the penalty and smoothness terms. Experimental results on real patient datasets show our method's superior performance compared to two similar methods.
\end{abstract}

\section{Introduction}

Segmentation of the left ventricle (LV) from dynamic contrast enhanced (DCE) magnetic resonance (MR) images of the heart is important for the analysis of cardiac functions. As an effective non-invasive imaging protocol, MRI has acquired great importance. Except for images with contrast enhancement, most images from the cardiac sequence show poor contrast between LV blood pool and myocardium wall. This, in addition to low spatial image resolution, makes segmentation of the LV a very challenging task when using only low level information (e.g. intensity, gradient,etc). This highlights the need for prior shape information in LV segmentation. In this paper we propose a graph cut based method to segment the LV blood pool and myocardium from DCE-MR image sequences using orientation histograms for prior shape information.

Previous works on segmentation using shape priors include, among others, active contours 1] and graph cuts 23. Graph cuts have the advantage of being fast, give globally optimal results and are not sensitive to initialization [4], while active contours are sensitive to initialization and can get trapped in local minima. Shape information in graphs is based on interaction between graph nodes (or image pixels). Inter-pixel interaction is generally limited to the immediate neighborhood although graph cuts can handle more complex neighborhoods [4].

G. Fichtinger, A. Martel, and T. Peters (Eds.): MICCAI 2011, Part III, LNCS 6893, pp. 420-427, 2011.

(C) Springer-Verlag Berlin Heidelberg 2011 
Therefore prior shape models assume great significance. We restrict further discussion to methods using prior shape information in graph cuts. The first works to use prior shape information in graph cuts were [2 3]. In [2] the zero level set function of a shape template of natural and medical images was used with the smoothness term to favor a segmentation close to the prior shape. Slabaugh et al. in [3] used an elliptical shape prior, under the assumption that many objects can be modeled as ellipses. However they employ many iterations where a preinitialized binary mask is updated to get the final segmentation. $\mathrm{Vu}$ et al. 5] use a discrete version of shape distance functions to segment multiple objects, which can be cumbersome. A flux-maximization approach was used in [6], while in [7] the smoothness cost was modified to include star shape priors.

Other methods have focused on segmenting the LV using statistical shape information. Mitchell et al. in 8] use a multistage active appearance model to segment LV and right ventricle (RV). Besbes et al. 9] used a control point representation of the LV prior and other images were deformed to match the shape prior. Shape knowledge has also been combined with dynamic information to account for cardiac shape variability [1011. Ali et al. in 12] construct a shape prior with some degree of variability and use it to segment DCE-MR kidney images.

We propose a method that has the capability to handle different shapes and uses a single image to get the shape prior. The novelties of our work are two-fold. First, we determine the shape penalty based on the distribution of orientation angles between a pixel and edge points on the prior shape. The angle distribution makes the metric robust and invariant to the number of points on a shape. Second, our method uses a single image from each dataset to get prior shape information. In Section 2 we describe our method in greater detail. Section 3 presents results of our experiments on real datasets, and we list our conclusions in Section 4 .

\section{Theory}

We use a second order Markov random field (MRF) energy function. MRFs are suitable for discrete labeling problems, and graph cuts can find their globally optimal solution for binary labels 4]. The energy function is given as

$$
E(L)=\sum_{s \in P} D\left(L_{s}\right)+\lambda \sum_{(s, t) \in N} V\left(L_{s}, L_{t}\right),
$$

where $P$ denotes the set of pixels, $L_{s}$ denotes label of pixel $s \in P$ and $N$ is the set of neighboring pixel pairs. $L_{s}$ denote the segmentation class of a pixel ( 0 for background and 1 for object). The labels of the entire set of pixels are denoted by $L . D\left(L_{s}\right)$, is a unary data penalty function derived from observed data and measures how well label $L_{s}$ fits pixel $s . V$ is a pairwise interaction potential that imposes smoothness and measures the cost of assigning labels $L_{s}$ and $L_{t}$ to neighboring pixels $s$ and $t . \lambda=0.01$ is a weight that determines the relative contribution of the two terms. Note that both $D$ and $V$ consist of two terms, each incorporating intensity and shape information. 
Overview of method: Our method has the following steps: 1) choose a reference image for each dataset and identify a small region inside and outside the LV. These regions give the reference intensity histograms of object and background. 2) Segment the image using only intensity information. This gives an oversegmented LV which is used to update the intensity distributions of object and background. The initial segmentation need not be optimal; and 3) incorporate shape information in $D$ and $V$ to get the final segmentation

Intensity Information: Patches are identified on the LV and background and their intensity distributions are modeled as Gaussians by estimating the mean and variance for each label. The intensity penalty $\left(D_{I}\right)$ is the negative log likelihood given by

$$
D_{I}(L)=-\log \operatorname{Pr}\left(I_{s} \mid L_{s}\right),
$$

where $I_{s}$ is the intensity at pixel $s, \operatorname{Pr}$ is the likelihood, and $L_{s}=$ object/background is the label. The intensity smoothness term $V_{I}$ assigns a low penalty at edge points based on the intensity of neighboring pixel pairs, and favours a piecewise constant segmentation result. It is defined as

$$
V_{I}\left(L_{s}, L_{t}\right)=\left\{\begin{array}{lr}
e^{-\frac{\left(I_{s}-I_{t}\right)^{2}}{2 \sigma^{2}}} \cdot \frac{1}{\|s-t\|}, L_{s} \neq L_{t} \\
0 & L_{s}=L_{t}
\end{array}\right.
$$

$\sigma$ determines the intensity difference up to which a region is considered as piecewise smooth. It is equal to the average intensity difference in a neighborhood w.r.t pixel $s$. $\|s-t\|$ is the Euclidean distance between $s$ and $t$.

Shape Information: A prior shape is used to incorporate shape knowledge into $D\left(L_{s}\right)$. In our method the shape penalty is determined at each pixel using only a single image from each dataset. Figure1 (g) shows an illustration of the reference shape as a continuous circle and different points $(A-F)$ inside and outside the shape. We shall explain the significance of the dotted line later. Figure 1 (h) shows the reference image from a typical dataset with the LV endocardium outlined in yellow and the epicardium outlined in green. First we explain how the shape prior is used to segment the LV blood pool. Later we extend our explanation to the segmentation of both blood pool and myocardium. Say for point $A$ (Fig. 1 (g)), we calculate the orientation angles of the line joining it to every point on the outline of the reference shape and plot the distribution of these angles. We notice that points inside and outside the reference shape have different distribution characteristics. The atan 2 function is used which returns angle values in the range $[-180,180]$.

All points outside the shape (i.e., points $A-B$ and $D-F$ ) have the orientation angles distributed over two or three quadrants of the angle space. The line joining $A$ to the closest point in the reference shape is the zero degree axis. For example, in the case of points outside the shape, the orientation angles are distributed over quadrants $I, I V$, or $I I, I I I$ (see Figs. 1 (a)-(b), (d)-(f)) while for point $C$ (which is inside the shape) the orientations are distributed over quadrants $I, I I, I I I, I \mathrm{~V}$ (Fig. 1 (c)). For histograms the horizontal axis shows the angle range from $-180^{\circ}$ 
to $+180^{\circ}$. For some points which are very close to the LV endocardium boundary but outside it, the distribution may be spread over three quadrants $(I, I I, I V$ or $I, I I I, I V)$ but never all the four quadrants. This discriminating feature acts as a basis for assigning penalty values to the pixel, i.e., if the angle distribution is spread over two or three quadrants then it is likely to belong to the background and therefore

$$
\begin{aligned}
& D_{S}\left(L_{s}=0\right)=0, \\
& D_{S}\left(L_{s}=1\right)=k_{1} .
\end{aligned}
$$

$D_{S}$ is the shape penalty; $k_{1}>1$ is a constant of high value so pixel $s$ does not take that label. If the distribution of angles is spread over four quadrants then the shape penalty is defined as

$$
\begin{aligned}
& D_{S}\left(L_{s}=0\right)=k_{1}, \\
& D_{S}\left(L_{s}=1\right)=0 .
\end{aligned}
$$

The above formulations makes the segmented shape very similar to the reference shape. Since the heart is made of muscular tissue there is bound to be elastic deformations in them with a resulting change in shape. In order to segment the deformed LV we relax the constraints on pixels near the prior's boundary. Referring back to Fig. 1 we observe that pixel $A$ is nearer to the reference shape than point $B$. We set a threshold normal distance of $d_{t h}$ pixels from the shape within which the LV may deform and allow for a change in labels of pixels within this $d_{t h}$ distance. Pixels which fall within this area are equally likely to take a particular label, and therefore have the same penalty. Therefore,

$$
D_{S}\left(L_{s}=0 / 1\right)=k_{2}, d_{s} \leq d_{t h},
$$

Here $0<k_{2}<k_{1}$, indicates that both labels are equally likely. $d_{s}$ refers to the distance of pixel $s$ from the shape. To determine $d_{t h}$ we adopt the following steps. After rigid alignment we choose 4 datasets (240 images) with large deformations (expecting it to cover a large range of shape deformations) and manually segment the endocardium region in all frames. For each dataset we compute the mean shape and distance of each shape from the mean. It is observed that the maximum distance of any individual shape from the average shape is $5 \mathrm{~mm}$ and the average distance is $3.5 \mathrm{~mm}$. Therefore we set $d_{t h}=5 \mathrm{~mm}$. Note that for any point lying on the edge of the prior shape $d_{s}=0$. For most such edge points the distribution of orientation angles may not be spread over four quadrants. But, since $d_{s}=0$, the penalty is assigned according to Eqn. (5).

Another contribution of our work is the formulation of the smoothness penalty based on the prior shape. Although the extent of interaction is usually limited to the immediate neighborhood, an arbitrary neighborhood can also be used 4]. For any pixel within $d_{t h}$ distance, it has some finite interaction with the closest pixel on the prior shape. This interaction is more important than the data penalty value to determine which pixels near the prior's boundary may change labels (due to deformations) as pixels within $d_{t h}$ distance of the contour have equal penalty for both labels. If such a pixel is very similar to the prior-shape's edge 
pixels (based on some feature), it is likely to have the same segmentation label. Thus we define $V_{S}$ as

$$
V_{S}\left(L_{s}, L_{t}\right)= \begin{cases}1-h, & L_{s} \neq L_{t} \\ 0 & L_{s}=L_{t}\end{cases}
$$

where $0<h \leq 1$ is the difference of orientation angle histograms given by the $\chi^{2}$ metric. The total energy function is thus defined as

$$
E(L)=\sum_{s \in P}\left[w_{1} D_{I}\left(L_{s}\right)+D_{S}\left(L_{s}\right)\right]+\lambda \sum_{(s, t) \in N}\left[V_{I}\left(L_{s}, L_{t}\right)+V_{S}\left(L_{s}, L_{t}\right)\right],
$$

$w_{1}=0.6$ decides the relative contribution of intensity information to the penalty and was set empirically.

Orientation histograms tend to overcome the shortest path problem inherent to graph cuts [7]. Therefore they are used in calculating $V_{S}$. To save further computation time the penalty values are calculated based on these orientation histograms without extra distance transform calculations. A single point in a bin is considered for spread in a quadrant. $V_{S}$ uses pixels within $d_{t h}$ distance of the prior while $V_{I}$ uses only neighboring pixels.

Extension to Multiple Classes: Segmentation of the image into LV blood pool, myocardium and background requires 3 labels. Those points which lie outside the continuous circle in Fig. 1] (g) but inside the dotted circle correspond to the myocardium. All points outside the dotted circle (points $G$ and $H$ ) are on

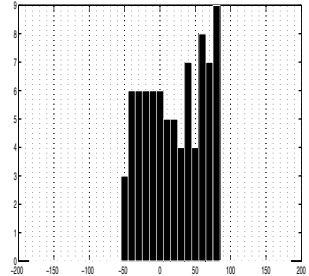

(a)

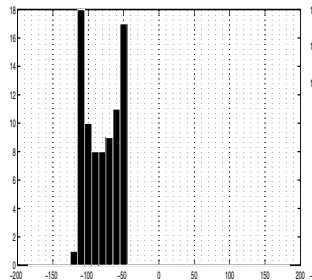

(e)

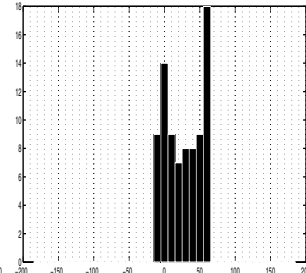

(b)

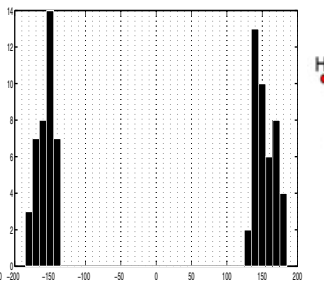

(f)

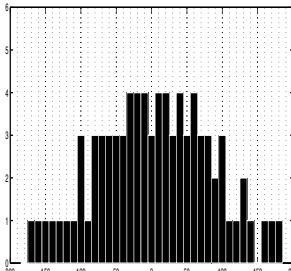

(c)

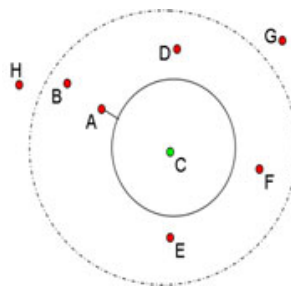

(g)

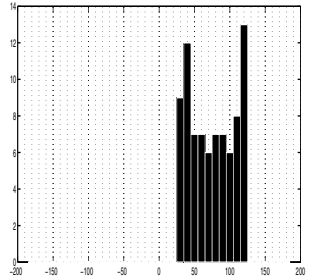

(d)

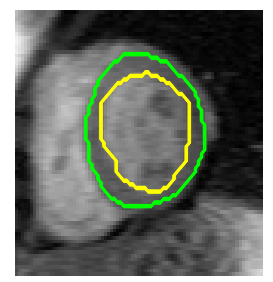

(h)

Fig. 1. Illustration of shape prior segmentation using orientation information. Orientation histograms for (a) point $A$; (b) point $B$; (c) point $C$; (d) point $D$; (e) point $E$; (f) point $F$; (g) synthetic image showing the different points outside and inside the shape; (h) reference cardiac image with LV endocardium highlighted in yellow and epicardium in green; 
the background. Depending upon the location of a pixel, different penalty values for each label is assigned. The value of $d_{t h}$ is the same as before.

\section{Experiments and Results}

Cardiac images were acquired on Siemens Sonata MR scanners following bolus injection of Gd-DTPA contrast agent. The pixel spacing ranges from $(1.5 \times 1.5)-$ $(2.8 \times 2.8) \mathrm{mm}^{2}$. The acquired datasets were all in $2 \mathrm{D}$ and a total of 7 datasets were used to test our method. Each dataset had 60 frames with a total of 420 images. The images were rigidly registered to the prior shape before segmentation. We have 3 segmentation labels for LV blood pool, LV myocardium and background. The LV blood pool and myocardium were manually segmented in all frames by experts. Automatic segmentations were obtained using four methods: graph cuts with intensity information alone $(G C)$; our method using shape priors with graph cuts $(G C S P)$; the methods in 26 . The automatic segmentations were compared with manual segmentation using Dice Metric $(D M)$ [13]. In all our experiments $k_{1}=10, k_{2}=1.2$ and $w_{1}=0.6$.

The method in 2] was implemented using the zero level set of the shape template in the smoothness cost for prior shape knowledge. For implementing [6] we calculate the appearance and location prior as described in their work, while for the shape prior we use the flux maximization and template based star shape constraint only. We weight the different components of the energy function to get the best segmentation results.

Figure 2 shows frames from different stages of contrast enhancement in a typical cardiac perfusion sequence alongwith the segmentation results from different methods. The outline of segmented masks are shown in different colours, and the manual segmentation is shown in red. These images exhibit poor contrast between the LV and the surrounding myocardium such that relying on intensity information alone leads to over-segmentation of the LV. The regions of inaccurate segmentation by each method is highlighted by yellow arrows. A visual inspection of the results indicates improved segmentation accuracy when using shape priors. Further, it also indicates that our method is more robust to shape changes. The formulation of our penalty and smoothness terms allows near-boundary pixels the flexibility to change their labels. If the reference shape is close to the average shape of the sequence, the accuracy does not change much. However if it has large deformation compared to other frames, lower $D M$ values are obtained.

Table 1 shows the quantitative segmentation performance for all four methods. Use of shape priors significantly improves the segmentation accuracy. Intensity information alone is not sufficient for LV segmentation in MR images. Our method shows highest $D M$ values and lowest contour distances, indicating that orientation histograms can very effectively account for shape changes due to deformations. Use of shape prior information in both penalty and smoothness term improves our method's accuracy over others. The average time taken for segmentation by different methods is $13 \mathrm{~s}(G C), 29 \mathrm{~s}(G C S P), 17 \mathrm{~s}[2]$ and $32 \mathrm{~s}$ 


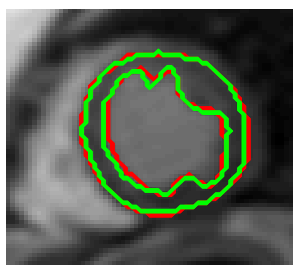

(a)

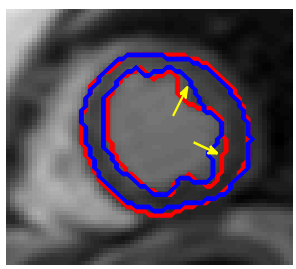

(b)

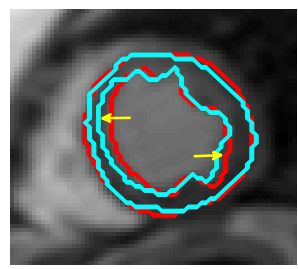

(c)

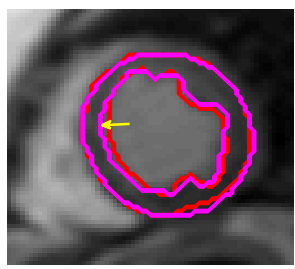

(d)

Fig. 2. Segmentation results using different methods. The manual segmentation is shown in red in all images and the results for automatic segmentation is shown in different colors. Results for (a) GCSP; (b) GC; (c) [2]; and (d) 6]. Areas of inaccurate segmentation are shown by yellow arrows.

Table 1. Comparative performance of segmentation accuracy using four methods and two metrics ( $D M$ and contour distance). The values show the average measures over all datasets. GC-graph cut with intensity only; GCSP is our method using graph cuts and shape priors; methods in [2], 6]. Values indicate the mean and standard deviation.

\begin{tabular}{|l|l|l|l|l||l|l|l|l|}
\hline & \multicolumn{4}{|c||}{ Dice Metric (\%) } & \multicolumn{4}{|c|}{ Mean Absolute Distance (mm) } \\
\hline & GC & GCSP & {$[2]$} & {$[6$} & GC & GCSP & {$[2]$} & {$[\underline{6}$} \\
\hline Epicardium & $87.1 \pm 1.2$ & $95.1 \pm 0.6$ & $92.8 \pm 1.3$ & $93.1 \pm 0.9$ & $3.2 \pm 0$. & $0.7 \pm 0.3$ & $1.4 \pm 0.4$ & $1.2 \pm 0.2$ \\
\hline Endocardium & $88.2 \pm 0.9$ & $94.7 \pm 1.1$ & $93.1 \pm 0.5$ & $92.4 \pm 1.0$ & $2.8 \pm 0.5$ & $0.8 \pm 0.4$ & $1.2 \pm 0.3$ & $1.3 \pm 0.4$ \\
\hline Overall LV & $87.7 \pm 1.1$ & $95.0 \pm 0.9$ & $92.9 \pm 0.8$ & $92 . \pm 0.9$ & $3.0 \pm 0.6$ & $0.7 \pm 0.3$ & $1.3 \pm 0.3$ & $1.3 \pm 0.3$ \\
\hline
\end{tabular}

for [6]. The time is for images of dimension $75-82 \times 83-87$ using MATLAB 7.5 on a PC with Pentium 4, $3 \mathrm{GHz}$ dual core processor. We did not optimize the code to take advantage of the multi core processor.

Influence of parameters $\boldsymbol{k}_{1}, \boldsymbol{k}_{2}, \boldsymbol{w}_{\mathbf{1}} \cdot w_{1}$ decides the relative contribution of intensity to the total penalty. If $w_{1}>0.9$ then $D_{I}$ 's contribution is high compared to $D_{S}$ and the final labels are influenced solely by $D_{I}$. On the other hand if $w_{1}<0.3 D_{S}$ dominates and $D_{I}$ has no influence on the final labels. $w_{1}=0.6$ is empirically chosen to give the best results for a wide range of datasets. $k_{1}$ should have value sufficiently higher than zero to impose hard constraints for labeling. $k_{2}$ should be low enough to not dominate over $V_{S}$, and also be greater than zero to ensure unambiguous solutions. If $k_{2}>1.5$ the contribution of $V_{S}$ towards the final labels decreases.

\section{Conclusion}

In this paper we have proposed a novel shape prior segmentation method using graph cuts for segmenting the LV from a sequence of dynamic cardiac perfusion images. Manual intervention is limited to identifying the LV in a reference image to get the prior shape. The shape penalty is calculated using the distribution of orientation angles from every pixel to the edge points of the prior shape. 
Penalty and smoothness terms are formulated such that pixels near the boundary of the shape prior can change their labels to account for shape change due to deformations. When combined with the intensity distributions of the object and background, our method results in accurate segmentation of the LV for low contrast perfusion MR images. Experimental results on real patient datasets show the advantages of using shape priors, and the superior performance of our method over two related methods. In future work we aim to extend the method for more segmentation classes and other imaging modalities.

\section{References}

1. Cremers, D., Tischhauser, F., Weickert, J., Schnorr, C.: Diffusion snakes: Introducing statistical shape knowledge into the Mumford-Shah functional. Intl. J. Comp. Vis. 50(3), 295-313 (2002)

2. Freedman, D., Zhang, T.: Interactive graph cut based segmentation with shape priors. In: IEEE CVPR, pp. 755-762 (2005)

3. Slabaugh, G., Unal, G.: Graph cuts segmentation using an elliptical shape prior. In: IEEE ICIP, pp. 1222-1225 (2005)

4. Boykov, Y., Veksler, O.: Fast approximate energy minimization via graph cuts. IEEE Trans. Pattern Anal. Mach. Intell. 23, 1222-1239 (2001)

5. Vu, N., Manjunath, B.: Shape prior segmentation of multiple objects with graph cuts. In: IEEE CVPR, pp. 1-8 (2008)

6. Chittajallu, D., Shah, S., Kakadiaris, I.: A shape driven mrf model for the segmentation of organs in medical images. In: IEEE CVPR, pp. 3233-3240 (2010)

7. Veksler, O.: Star shape prior for graph cut segmentation. In: Forsyth, D., Torr, P., Zisserman, A. (eds.) ECCV 2008, Part III. LNCS, vol. 5304, pp. 454-467. Springer, Heidelberg (2008)

8. Mitchell, S., Lelieveldt, B., van der Geest, R., Bosch, H., Reiver, J., Sonka, M.: Multistage hybrid active appearance models: Segmentation of cardiac $\mathrm{mr}$ and ultrasound images. IEEE Trans. Med. Imag 20(5), 415-423 (2001)

9. Besbes, A., Komodakis, N., Paragios, N.: Graph-based knowledge-driven discrete segmentation of the left ventricle. In: IEEE ISBI, pp. 49-52 (2009)

10. Zhu, Y., Papademetris, X., Sinusas, A., Duncan, J.: Segmentation of left ventricle from $3 \mathrm{~d}$ cardiac $\mathrm{mr}$ image sequence using a subject specific dynamic model. In: Proc. IEEE CVPR, pp. 1-8 (2008)

11. Sun, W., Çetin, M., Chan, R., Reddy, V., Holmvang, G., Chandar, V., Willsky, A.: Segmenting and tracking of the left ventricle by learning the dynamics in cardiac images. In: Christensen, G.E., Sonka, M. (eds.) IPMI 2005. LNCS, vol. 3565, pp. 553-565. Springer, Heidelberg (2005)

12. Ali, A., Farag, A., El-Baz, A.: Graph cuts framework for kidney segmentation with prior shape constraints. In: Ayache, N., Ourselin, S., Maeder, A. (eds.) MICCAI 2007, Part I. LNCS, vol. 4791, pp. 384-392. Springer, Heidelberg (2007)

13. Pluempitiwiriyawej, C., Moura, J., Wu, Y., Ho, C.: STACS: new active contour scheme for cardiac MR image segmentation. IEEE Trans. Med. Imag. 24(5), 593603 (2005) 